\title{
Intracutaneous Delivery of Gelatins Reduces Fat Accumulation in Subcutaneous Adipose Tissue
}

\author{
Sung-Min $\mathrm{An}^{\dagger}$, Min Jae Kim${ }^{\dagger}$, Keum-Yong Seong, Jea Sic Jeong, Hyeon-Gu Kang, So Young Kim, \\ Da Som Kim, Da Hee Kang, Seung Yun Yang and Beum-Soo An \\ Department of Biomaterials Science, Life and Industry Convergence Institute, Pusan National University, Miryang, \\ Korea
}

\begin{abstract}
Subcutaneous adipose tissue (SAT) accumulation is a constitutional disorder resulting from metabolic syndrome. Although surgical and non-surgical methods for reducing SAT exist, patients remain non-compliant because of potential adverse effects and cost. In this study, we developed a new minimally-invasive approach to achieve SAT reduction, using a microneedle $(\mathrm{MN})$ patch prepared from gelatin, which is capable of regulating fat metabolism. Four gelatin types were used: three derived from fish (SA-FG, GT-FG 220, and GT-FG 250), and one from swine (SM-PG 280). We applied gelatin-based MN patches five times over 4 weeks to rats with high-fat diet (HD)induced obesity, and determined the resulting amount of SAT. We also investigated the histological features and determined the expression levels of fat metabolism-associated genes in SAT using hematoxylin and eosin staining and western blotting, respectively. SAT decreased following treatment with all four gelatin MN patches. Smaller adipocytes were observed in the regions treated with SA-FG, GT-FG 250, and SM-PG 280 MNs, demonstrating a decline in fat accumulation. The expression levels of fat metabolism-associated genes in the MN-treated SAT revealed that GT-FG 220 regulates fatty acid synthase (FASN) protein levels. These findings suggest that gelatin $\mathrm{MN}$ patches aid in decreasing the quantity of unwanted SAT by altering lipid metabolism and fat deposition.
\end{abstract}

\section{Key words: Subcutaneous adipose tissue, Gelatin, Microneedle, Obesity}

\section{INTRODUCTION}

Obesity, which is increasing globally, is defined as an excess of body fat mass resulting from body fat accumulation over time following excess energy intake or low energy expenditure (1). It is closely linked to several health risks, and leads to insulin resistance (IR) and the development of type 2 diabetes (2). It equally results in hypertension and certain cancers $(3,4)$. Adipose tissue (AT) is the main organ for energy storage. It can be classified into two broad divisions: subcutaneous AT (SAT) and visceral AT (VAT). SAT is specifically responsible for storing over $80 \%$ of the total fat in the body and is mainly deposited in the abdominal and gluteal-femoral regions (5).

The application of SAT reduction for cosmetic purposes (improve body shape and image), has attracted much
Correspondence to: Beum-Soo An, Department of Biomaterials Science, Life and Industry Convergence Institute, Pusan National University, 1268-50, Samnangjin-ro, Samnangjin-eup, Miryang-si, Gyeongsangnam-do 50463, Korea

E-mail: anbs@pusan.ac.kr

${ }^{\dagger}$ The first two authors contributed equally to this work.

This is an Open-Access article distributed under the terms of the Creative Commons Attribution Non-Commercial License (http:// creativecommons.org/licenses/by-nc/3.0) which permits unrestricted non-commercial use, distribution, and reproduction in any medium, provided the original work is properly cited.
Abbreviations: ANOVA, Analysis of variance; AT, Adipose tissue; CNC, Computer numerical control; FASN, Fatty acid synthase; FDA, Food and Drug Administration; HD, High-fat diet; H\&E, Hematoxylin and eosin; HIFU, High-intensity focused ultrasound; HRP, Horseradish peroxidase; IR, Insulin resistance; MN, Microneedle; PBS, Phosphate-buffered saline; PBS-T, PBS with $0.05 \%$ Tween-20; PDMS, Polydimethylsiloxane; PNU-IACUC, Pusan National University Institutional Animal Care and Use Committee; PPARG, Peroxisome proliferator-activated receptor- $\gamma$; PPC, Phosphatidylcholine; RF, Radiofrequency; SAT, Subcutaneous adipose tissue; SD, Sprague Dawley; SREBP-1c, Sterol regulatory element-binding protein 1c; SEM, Standard error of the mean; VAT, Visceral adipose tissue; VLDL, Very low density lipoprotein. 
attention (6). Recent reports indicate that SAT is significantly associated with metabolic syndrome and its characteristics, independent of age and sex (7). Moreover, excess SAT causes considerable problems linked to the development of AT dysfunction and fat accumulation in ectopic depots such as the liver and muscle tissue (8-10). For these reasons, various chronic diseases such as liver cirrhosis and certain cancers are closely associated with fat metabolism (11).

Surgical and non-surgical methods are used for SAT reduction $(12,13)$. Specifically, the intralesional injection of chemical substances such as phosphatidylcholine (PPC), which cause the lysis of AT, is effective in diminishing localized fat accumulation (14). However, this method has several side effects such as pain, erythema, and an infectious granulomatous reaction $(15,16)$. Alternatively, several natural polymers including low-molecular-weight chitosan, gelatin, and chondroitin sulfate have been used as dietary supplements to accelerate fat reduction through several pathways (17-19). Gelatin is an inexpensive alternative, readily available in nature, and widely used in diverse biomedical applications. In a previous study, we demonstrated that gelatin suppresses lipogenesis and accelerates lipolysis in adipocytes and adipose tissues (20). Although these natural polymers are safe and effective as dietary supplements, information on their local effects on unwanted SAT is limited.

Recently, non-invasive procedures such as the use of lasers, high-intensity focused ultrasound (HIFU), cryolipolysis, and radiofrequency (RF) therapy, which have few side effects and increased patient compliance, have been examined for local fat reduction (21-23). However, these techniques need additional expensive consumable devices, which require trained clinicians for safe operation. Consequently, a new SAT reduction procedure that is effective, minimally-invasive, inexpensive, self-administrable, and does not require additional devices is needed.

To develop an appropriate platform for SAT reduction, we manufactured a dissolving microneedle (MN) patch from a biodegradable natural polymer (gelatin), which has anti-obesity effects. In this study, we confirmed the antiobesity effects of gelatin and developed a novel therapy for SAT reduction in desired regions of the body based on the application of MNs. We also verified the effects of gelatin MNs on fat metabolism in the SAT of rats with highfat diet (HD)-induced obesity.

\section{MATERIALS AND METHODS}

Materials. Anti- $\beta$-actin antibodies were purchased from Cell Signaling Technology, Inc (Beverly, MA, USA). Anti-fatty acid synthase (anti-FASN), anti-sterol regulatory element-binding protein 1c (anti-SREBP-1c), and antiperoxisome proliferator-activated receptor- $\gamma$ (anti-PPARG) antibodies as well as horseradish peroxidase (HRP)-conjugated anti-rabbit and anti-mouse IgG antibodies were purchased from Santa Cruz Biotechnology, Inc (Santa Cruz, CA, USA). Cold water fish skin gelatin was purchased from Sigma Aldrich (St. Louis, MO, USA); porcine skin gelatin from Sammi Co., Ltd. (Anyang, Korea); fish skin gelatin from Gel Tec Co., Ltd. (Busan, Korea); and polydimethylsiloxane (PDMS; Sylgard 184) from Dow Corning (Midland, MI, USA).

Preparation of biopolymer-based MN patches. MN patches were prepared based on a previously described protocol (24). To fabricate the gelatin MNs, we prepared a $10 \% \mathrm{w} / \mathrm{w}$ gelatin solution in a $40^{\circ} \mathrm{C}$ water bath. The PDMS molds for fabricating the gelatin $\mathrm{MN}$ patches were replicated from a positive bullet-shaped MN array milled using a computer numerical control (CNC) machine. A gelatin MN patch $\left(1 \times 1 \mathrm{~cm}^{2}\right)$ was prepared by solvent-casting the gelatin solution in a vacuum chamber maintained at $60^{\circ} \mathrm{C}$. Each gelatin $\mathrm{MN}$ patch was dried at $60^{\circ} \mathrm{C}$ for $12 \mathrm{hr}$. After cooling to room temperature, the gelatin $\mathrm{MN}$ patches were gently peeled from the PDMS molds.

Preparation of animals. The animal care protocol used in the present study was reviewed and approved by the Pusan National University Institutional Animal Care and Use Committee (PNU-IACUC), and was based on their ethical procedures and scientific care guidelines. Thirty male Sprague Dawley (SD) rats $(160-180 \mathrm{~g}$ ) for the experiments were purchased from Samtako (Osan, Korea), and transferred to the Pusan National University Laboratory Animal Resources Center (Busan, Korea), accredited by the Korea Food and Drug Administration (KFDA), according to the National Institutes of Health guidelines. The rats were housed in cages maintained under a 12:12 hr light/dark cycle at a constant temperature of $23 \pm$ $1^{\circ} \mathrm{C}$. All experimental animal procedures were approved by the Ethics Committee of Pusan National University (Busan, Korea; approval no. PNU-2018-1836). After 3 days of acclimatization, the rats were fed a HD (D12492 Rodent Diet with $60 \mathrm{kcal} \%$ fat; Research Diets Inc., New Brunswick, NJ, USA). After 2 weeks on the HD, the resultant obese rats were subdivided into five groups (of 6 rats each): one control and four MN groups (Sigma Aldrichfish gelatin (SA-FG), Gel Tec-fish gelatin 220B and 250B (GT-FG 220 and 250), and Sammi-porcine gelatin BL280P (SM-PG 280)). Gelatin MN $\left(1 \times 1 \mathrm{~cm}^{2}\right)$ patches were used. The inguinal region of each rat was shaved, and the MN patches attached to the dorsal skin on one side every 3 days (the patches were attached a total of five times). Inguinal SAT located below the sites of interest (treated with MNs or untreated) and epididymal AT were removed, rinsed with phosphate-buffered saline (PBS) and prepared for further experiments. After applying the MNs, the AT was 
fixed in paraffin and stained with hematoxylin and eosin (H\&E) from Sigma-Aldrich. Images of the stained adipocytes were captured using an (Olympus, Tokyo, Japan).

Western blotting analysis. For western blotting, the adipocyte protein samples were extracted using Pro-prep solution from iNtRON Biotechnology (Seoul, Korea), according to the manufacturer's instructions. Proteins were separated by sodium dodecyl sulfate-polyacrylamide gel electrophoresis (SDS-PAGE; 8-12\% SDS), and transferred to nitrocellulose membranes; from Daeil Lab Service Co., Ltd (Seoul, Korea). The membranes were blocked for $2 \mathrm{hr}$ with $5 \%$ Difco $^{\mathrm{TM}}$ skimmed milk (Sparks, MD, USA) in PBS with $0.05 \%$ Tween-20 (PBS-T). After blocking, the membranes were incubated overnight with anti-FASN rabbit (diluted 1:1000), anti-SREBP-1c mouse (diluted 1:1000), and anti-PPARG mouse (diluted 1:1000) antibodies (Santa Cruz Biotechnology, Inc.), then further incubated for $1 \mathrm{hr}$ with HRP-conjugated anti-rabbit or antimouse antibodies (diluted 1:2000) in 5\% skimmed milk in PBS-T. Luminol reagent from Bio-Rad (Hercules, CA, USA) was used to visualize antibody binding. Each blot was stripped by incubation with $2 \%$ SDS and $100 \mathrm{mM} 2-$ mercaptoethanol in $62.5 \mathrm{mM}$ Tris- $\mathrm{HCl}$ ( $\mathrm{pH} \mathrm{6.8)}$ ) for $30 \mathrm{~min}$ at $50-60^{\circ} \mathrm{C}$. The membranes were subsequently probed with a $\beta$-actin antibody (diluted 1:3000) (Santa Cruz Biotechnology, Inc.), as an internal control. The blots were scanned using Gel Doc 1000 version 1.5 (Bio-Rad), and the band intensities were normalized to the $\beta$-actin levels.

Histological analyses. The recovered SAT was fixed with $10 \%$ formalin, embedded in paraffin wax, and sectioned into $4-\mu \mathrm{m}$-thick slices. The SAT sections were deparaffinized, rehydrated through graded alcohol using standard procedures, and then stained with H\&E. Tissue images were captured at $40 \times$ magnification, using a model BX50F-3 optical microscope from Olympus (Tokyo, Japan), and histological observations made. We examined five representative images per tissue section for the relevant calculations, and used at least three rats. Briefly, cross-sectional areas of the adipocytes were obtained from perimeter tracings using Image $\mathrm{J}$ software (version 1.45x; National Institutes of Health, Bethesda, MD, USA).

Statistical analyses. Results are presented as the mean \pm standard error of the mean (SEM). Data were investigated using one-way analysis of variance (ANOVA) (SPSS for Windows, 10.10, standard version) from SPSS Inc (Chicago, IL, USA). $P$-values $<0.05$ indicate statistically significant differences.

\section{RESULTS}

Effects of gelatin MNs on body weight and food intake in rats with HD-induced obesity. We used rats with HD-induced obesity to determine the effects of the gelatin
(A)
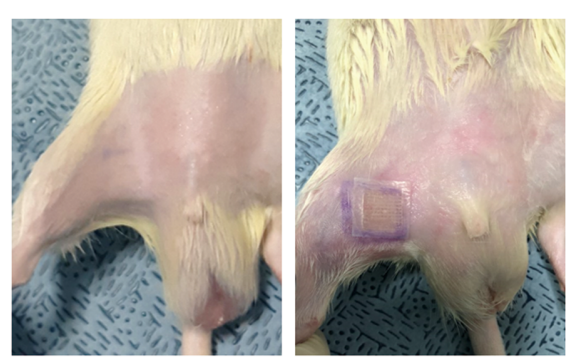

(C)

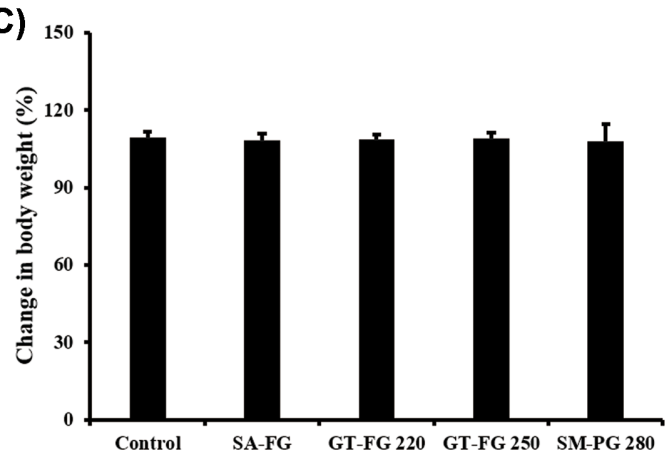

(B)

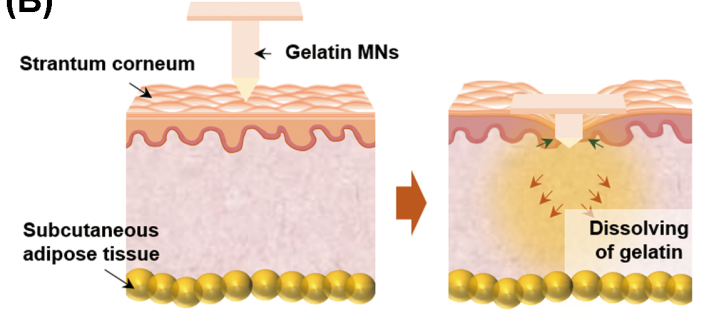

(D)

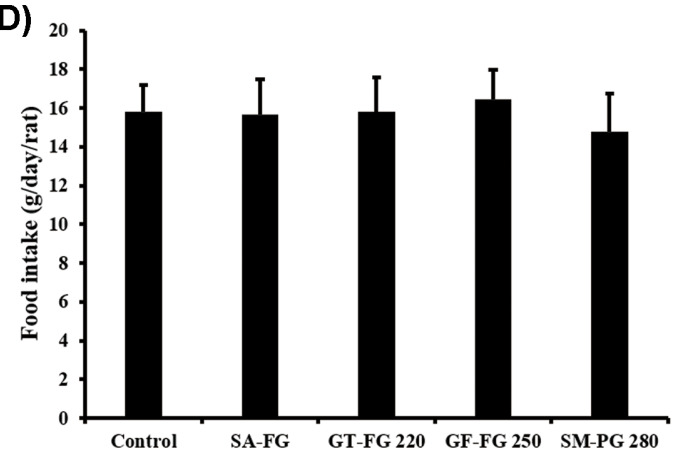

Fig. 1. Effect of microneedles (MNs) on body weight and food intake in rats with high-fat diet (HD)-induced obesity. MN gelatin patches were applied to the inguinal skin of rats with HD-induced obesity. Representative images of in vivo study displaying the MN application process (A). Schematic illustration of MNs applied to the inguinal skin (B). Changes in body weight and food intake in rats with $\mathrm{HD}$-induced obesity (C-D). Data are expressed as mean \pm SEM of five rats/group. 
MNs. The gelatin MNs were applied to the inguinal region, which is a known site of SAT accumulation (Fig. 1A). The gelatin MNs were grouped by species of gelatin origin (fish and porcine) and manufacturing company. The prepared MNs were only applied on one side of the inguinal region in rats with HD-induced obesity. The fabricated gelatin MN patches (Fig. 1B) consisted of $1 \times 1 \mathrm{~cm}^{2}$ bullet-shaped needles with a base width of $250 \mu \mathrm{m}$ and a height of $750 \mu \mathrm{m}$. The gelatin MN patches were designed to be dissolvable, and increase the local release of polymers at the targeted region. The MN patches were applied every 3 days for 4 weeks (the patches were attached a total of five times). Body weight and food intake were measured every 3 days throughout the application period and normalized with the values obtained on the first day of application. Fig. 1C shows the alterations in body weight, which were non-significant. Additionally, food intake among the groups was not significantly different (Fig. 1D).

Effects of gelatin MNs on the weight of SAT in rats with HD-induced obesity. To determine whether there was locally specific SAT reduction in the regions to which the MN patches were applied, we excised and weighed the inguinal SAT and epididymal AT post-experiment. The SAT weights were normalized to the epididymal AT weights. Fig. 2A reveals that the inguinal SAT masses where the $\mathrm{MN}$ patches were attached (left side) were greatly reduced compared with the regions without treatment (right side) in the GT-FG 250 and SM-PG 280 groups. Furthermore, the normalized SAT weights of the GT-FG 250 and SMPG 280 groups were significantly reduced compared to the weights in the control group (Fig. 2B).

\section{(A)}

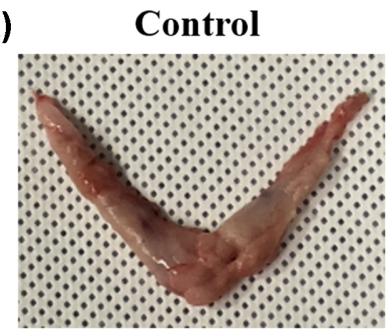

SA-FG

GT-FG 220

GT-FG 250
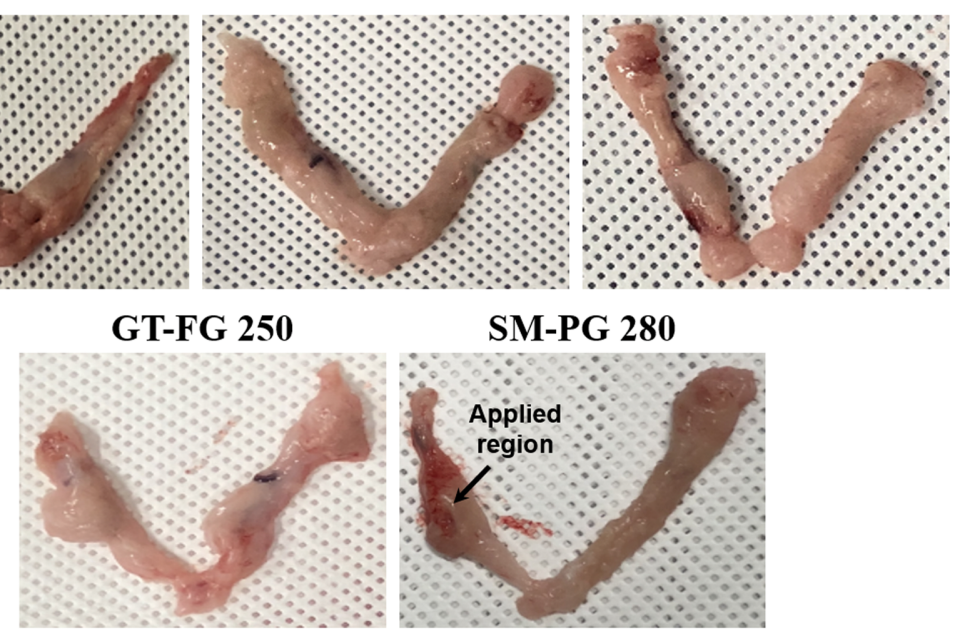

SM-PG 280

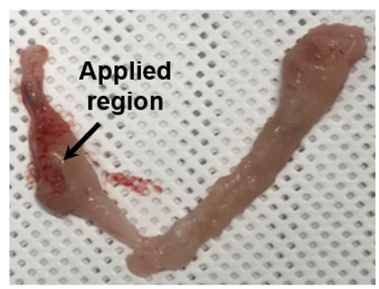

(B)

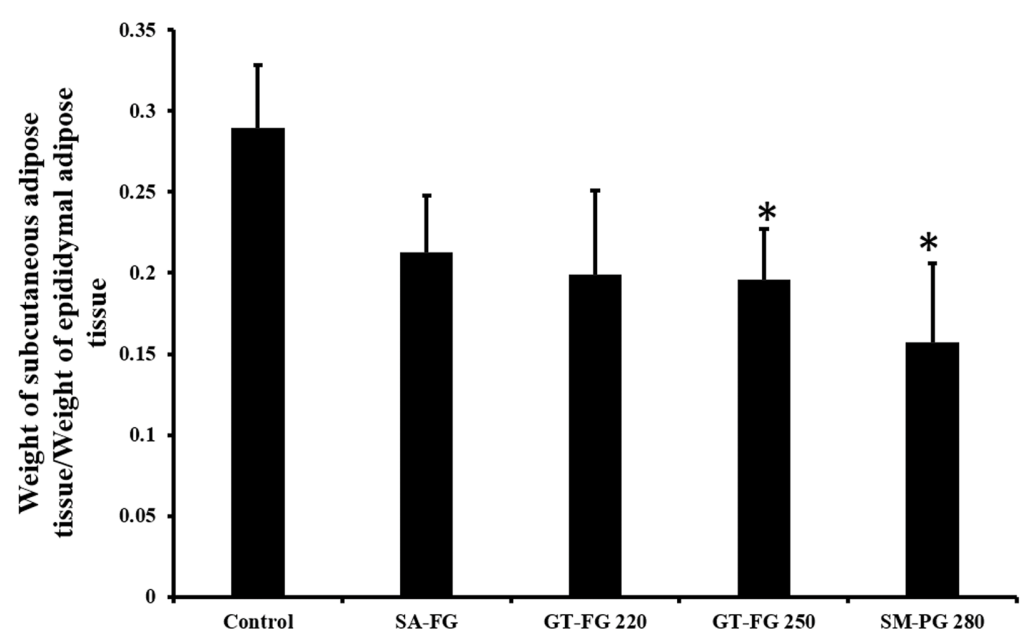

Fig. 2. Effect of microneedles (MNs) on the shape and weight of subcutaneous adipose tissue (SAT) in rats with high-fat diet (HD)induced obesity. Images of SAT post MN patch application to the inguinal skin (A). The arrow indicates the SAT treated with thegelatin MN patch. SAT weight was normalized to the weight of the epididymal AT (B). Data are expressed as mean \pm SEMs of five rats/ group. ${ }^{*} p<0.05$ compared with the control group. 
(A)

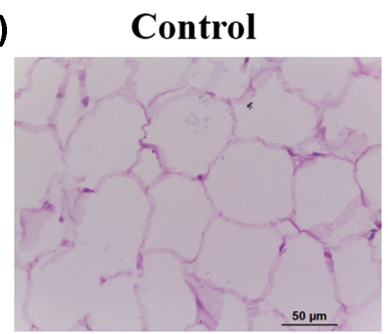

SA-FG

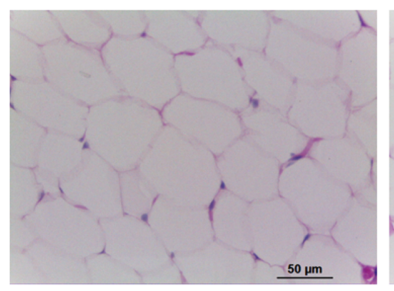

GT-FG 220

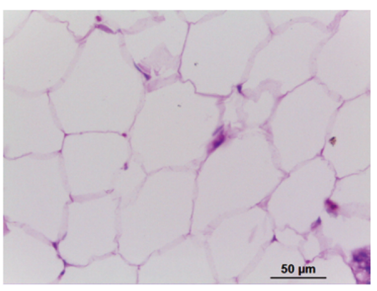

GT-FG 250

SM-PG 280
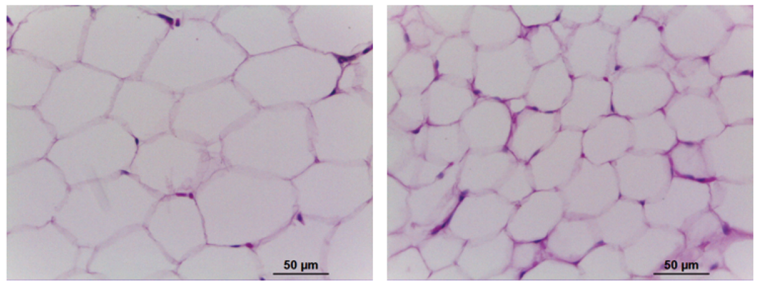

(B)

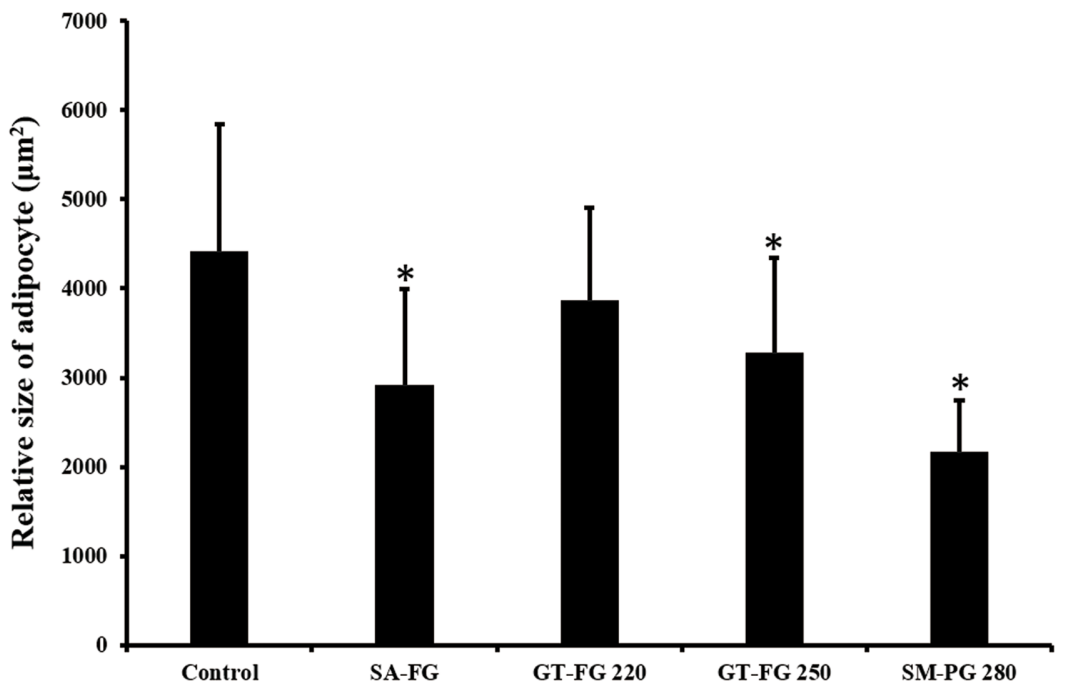

Fig. 3. Histological images of subcutaneous fat tissues post application of gelatin microneedle (MN) patches. Subcutaneous adipose tissue (SAT) treated with MN patches was stained with hematoxylin and eosin (H\&E) (A). The relative sizes of the adipocytes were measured and calculated (B). Data are expressed as mean \pm SEMs of adipocyte sizes from five images of three rats/group. ${ }^{*} p<0.05$ compared with the control group.

\section{Changes in SAT deposits following application of the} gelatin MN patches. We also examined the histological alteration in SAT following application of the gelatin MN patches, to determine how the patches change adipocyte morphology (Fig. 3). After applying the MN patches, we fixed the treated inguinal SAT in paraffin and stained it with H\&E.

The diameters of the lipid droplets in the SA-FG group, the GT-FG 250 group, and especially the SM-PG 280 group, were smaller than those in the control group, representing a reduction in fat accumulation (Fig. 3A). The results of the changes in adipocyte size are shown in Fig. $3 \mathrm{~B}$. These results suggest that gelatin MNs (especially SM-PG $280 \mathrm{MNs}$ ) reduce fat accumulation, regardless of changes in body weight.
Effect of gelatin MNs on the expression of adipogenic and lipogenic genes in SAT. To determine how gelatin MNs regulate fat metabolism, we used western blotting to measure the expression levels of genes involved in adipogenesis and lipogenesis (Fig. 4). We determined protein expression levels of the lipogenic enzyme FASN and its transcriptional regulators SREBP-1c and PPARG in SAT. Representative protein expression bands attributable to FASN, SREBP-1c, and PPARG are shown in Fig. 4A. FASN (lipogenic enzyme) levels were significantly reduced (up to $80 \%$ reduction) in the SAT of the GT-FG 220 group (Fig. 4B). However, SREBP-1c and PPARG expression levels in SAT were not altered following the application of any of the gelatin MN patches (Fig. 4C). SM-PG 280 MNs reduced the expression of PPARG, 
(A)

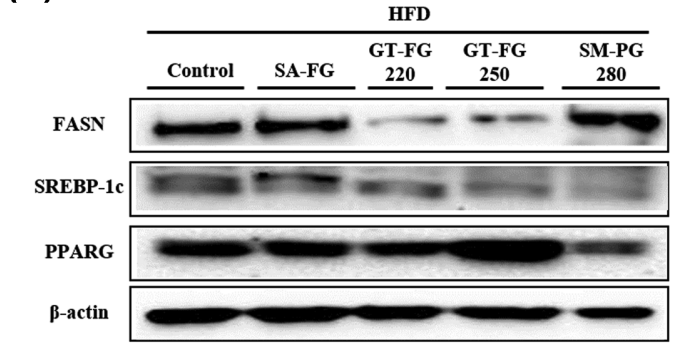

(C)

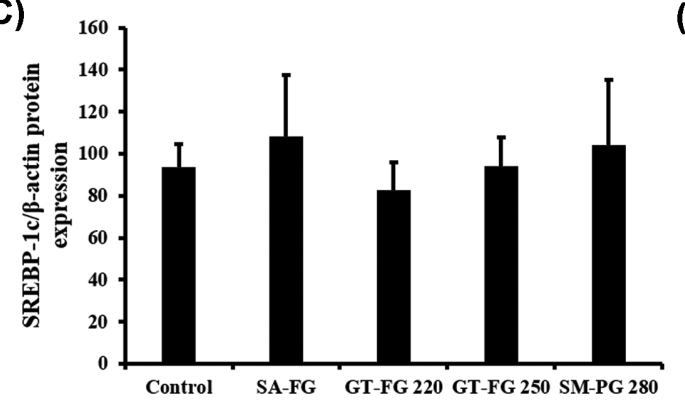

(B)

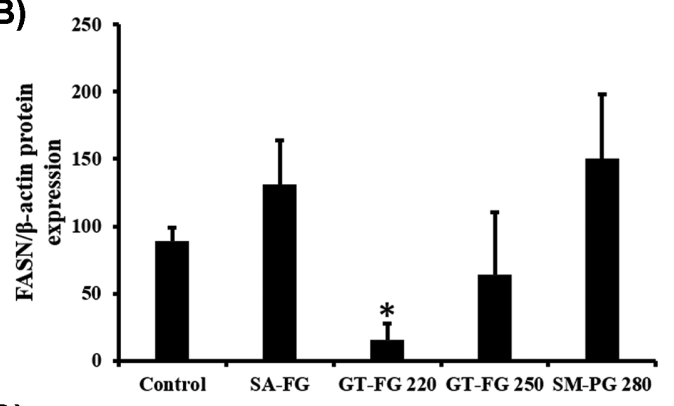

(D)

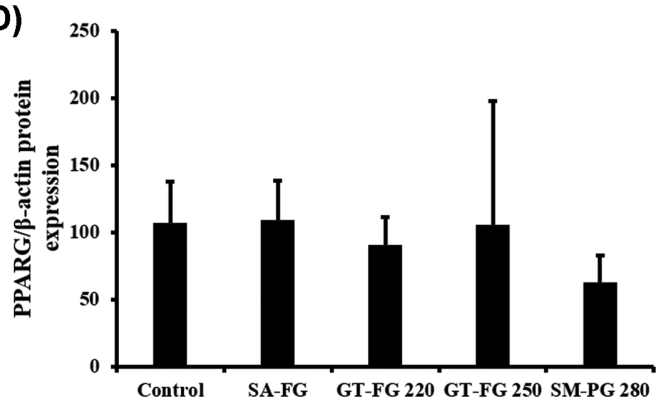

Fig. 4. Effects of microneedles (MNs) on the protein expression of adipogenic and lipogenic genes in the subcutaneous adipose tissue (SAT) of rats with high-fat diet (HD)-induced obesity. The inguinal skin of the rats were treated with MNs. Representative blot images of protein expressions are shown (A). The protein levels of fatty acid synthase (FASN) (B), sterol regulatory element-binding protein 1c (SREBP-1c) (C), and anti-peroxisome proliferator-activated receptor- $\gamma$ (PPARG) (D) were determined by western blotting. $\beta$ Actin expression in each sample was used as an internal control to normalize expression. The results shown are representative of at least three independent experiments. Data are expressed as mean \pm SEMs of protein levels of tissues from $\geq 3$ rats/group. ${ }^{*} p<0.05$ compared with the control group.

which facilitates lipogenesis, although the reduction was not significant (Fig. 4D).

\section{DISCUSSION}

Recently, obesity resulting from fat accumulation in local regions has become a cosmetic problem with social and psychological ramifications. It is generally associated with the enlargement of SAT, which is mainly distributed on the hips and thighs (25). Hypertrophic SAT sometimes contributes to the pathogenesis of metabolic diseases, as seen in animal models of obesity and obese individuals $(26,27)$. To ameliorate the excessive accumulation of SAT, patients usually undergo surgical procedures including liposuction, which has a number of potential risks including pain, infection, numbness, and/or scarring (25). Therefore, there has been a recent focus on developing an alternative approach to reducing SAT. MNs are micronscale needle-like structures no more than $1 \mathrm{~mm}$ in length, used to pierce the upper layer of the skin to enable transdermal drug delivery (28). In several previous studies, solid MNs have been used with RF delivery devices for the superficial penetration of the skin (29). In the case of gelatin MNs, the applied MN patch creates micropunctures which significantly improve the permeation of the gelatin content into the skin.
The anti-obesity effect of gelatin, the natural polymer investigated in the present study, has not been studied extensively. A previously study reported that oral gelatin treatment reduced plasma cholesterol and triglyceride levels in hypercholesterolemic rats (30). However, orally administered gelatin can be metabolized through various pathways in the intestine and liver, suggesting that there are limitations to the effectiveness of this orally administered gelatin. In a previous study, in investigating the effect of a single application of a gelatin $\mathrm{MN}$ patch to the dorsal skin, known to contain brown AT, we demonstrated that single attachment of an MN patch to the dorsal skin significantly reduced $(60 \%)$ regional SAT without altering the total weight (20). This proved the necessity of verifying the anti-obesity effect of gelatin MNs in white AT. Consequently, in the present study, we conducted animal experiments by dissolving $\mathrm{MN}$ patches in the inguinal region, which contains more white AT than any other body part. We also carried out the experiments over a relatively long period (4 weeks), and applied the MN patches every 3 days. MNs were prepared using gelatin from various sources (e.g., fish and porcine) and manufacturing companies to determine which gelatin has optimum anti-obesity effects.

The normalized SAT weights of the gelatin MN groups, especially the GT-FG 250 and SM-PG 280 groups, were 
significantly reduced, compared with the control group. However, the body weight of the rats in all groups was not significantly altered, although the SAT was reduced. We assumed that SAT occupied a very small portion of body weight, therefore, the change of SAT does not affect body weight in statistical analysis.

Furthermore, the gelatin MNs derived from fish (SA-FG group and GT-FG 250 group) and swine (SM-PG 280 group) significantly reduced fat deposition in the inguinal adipocytes. The accumulation of fat is associated with the levels of triglycerides, a form of fat stored in the body (31). Previous authors reported that glycine, which constitutes $22 \%$ of the amino acids in gelatin, reduces the size of adipocytes and the secretion of hepatic very low density lipoprotein (VLDL) in obese animals (32).

To corroborate this suggestion, we measured the protein expression levels of genes involved in lipogenesis and adipogenesis, to determine how MNs regulate fat metabolism. We determined the protein expression levels of two transcription factors related to lipogenesis (SREBP-1c and PPARG) and their target gene FASN (33-35). The GT-FG $220 \mathrm{MNs}$ significantly reduced FASN protein expression, whereas the expression levels of SREBP-1c and PPARG were not significantly affected by any of the $\mathrm{MN}$ patches tested. These gene expression patterns contradicted the results of the histological analyses and those of our previous study, which demonstrated significant adipogenesisrelated gene regulation (20), which may be attributed to long-term MN application. The repeated MN application $(5 \times)$ may have reduced the responsiveness to gelatin in the SAT with respect to the expression of lipogenic genes.

In summary, our results demonstrate that the application of gelatin MNs, especially porcine-derived MNs (SM-PG 280 group), reduces fat metabolism and SAT accumulation in obese rats. These data therefore suggest the use of biodegradable gelatin MNs for targeted SAT reduction, which has minimal side effects.

\section{ACKNOWLEDGMENTS}

This work was supported by a 2-year research grant from Pusan National University.

\section{CONFLICT OF INTEREST}

The authors declare no conflicts of interest.

Received December 11, 2018; Revised February 21, 2019; Accepted April 25, 2019

\section{REFERENCES}

1. Cheung, W.W. and Mao, P. (2012) Recent advances in obesity: genetics and beyond. ISRN Endocrinol., 2012, 536905.
2. Bell, J.A., Kivimaki, M. and Hamer, M. (2014) Metabolically healthy obesity and risk of incident type 2 diabetes: a meta-analysis of prospective cohort studies. Obes. Rev., 15, 504-515.

3. Coutinho, P.M., Deleury, E., Davies, G.J. and Henrissat, B. (2003) An evolving hierarchical family classification for glycosyltransferases. J. Mol. Biol., 328, 307-317.

4. Morigny, P., Houssier, M., Mouisel, E. and Langin, D. (2016) Adipocyte lipolysis and insulin resistance. Biochimie, 125, 259-266.

5. Wajchenberg, B.L. (2000) Subcutaneous and visceral adipose tissue: their relation to the metabolic syndrome. Endocr. Rev., 21, 697-738.

6. Friedmann, D.P. (2015) A review of the aesthetic treatment of abdominal subcutaneous adipose tissue: background, implications, and therapeutic options. Dermatol. Surg., 41, 18-34.

7. Carr, D.B., Utzschneider, K.M., Hull, R.L., Kodama, K., Retzlaff, B.M., Brunzell, J.D., Shofer, J.B., Fish, B.E., Knopp, R.H. and Kahn, S.E. (2004) Intra-abdominal fat is a major determinant of the National Cholesterol Education Program Adult Treatment Panel III criteria for the metabolic syndrome. Diabetes, 53, 2087-2094.

8. Bluher, M. (2013) Adipose tissue dysfunction contributes to obesity related metabolic diseases. Best Pract. Res. Clin. Endocrinol. Metab., 27, 163-177.

9. Rasouli, N., Molavi, B., Elbein, S.C. and Kern, P.A. (2007) Ectopic fat accumulation and metabolic syndrome. Diabetes Obes. Metab., 9, 1-10.

10. Kloting, N. and Bluher, M. (2014) Adipocyte dysfunction, inflammation and metabolic syndrome. Rev. Endocr. Metab. Disord., 15, 277-287.

11. Ma, X., Lee, P., Chisholm, D.J. and James, D.E. (2015) Control of adipocyte differentiation in different fat depots; implications for pathophysiology or therapy. Front. Endocrinol., 6, 1 .

12. Kennedy, J., Verne, S., Griffith, R., Falto-Aizpurua, L. and Nouri, K. (2015) Non-invasive subcutaneous fat reduction: a review. J. Eur. Acad. Dermatol. Venereol., 29, 1679-1688.

13. Derrick, C.D., Shridharani, S.M. and Broyles, J.M. (2015) The safety and efficacy of cryolipolysis: a systematic review of available literature. Aesthet. Surg. J., 35, 830-836.

14. Rittes, P.G. (2003) The use of phosphatidylcholine for correction of localized fat deposits. Aesthetic Plast. Surg., 27, 315-318.

15. Noh, Y. and Heo, C.Y. (2012) The effect of phosphatidylcholine and deoxycholate compound injections to the localized adipose tissue: an experimental study with a murine model. Arch. Plast. Surg., 39, 452-456.

16. Rotunda, A.M. and Kolodney, M.S. (2006) Mesotherapy and phosphatidylcholine injections: historical clarification and review. Dermatol. Surg., 32, 465-480.

17. Han, L.K., Sumiyoshi, M., Takeda, T., Chihara, H., Nishikiori, T., Tsujita, T., Kimura, Y. and Okuda, H. (2000) Inhibitory effects of chondroitin sulfate prepared from salmon nasal cartilage on fat storage in mice fed a high-fat diet. Int. J. Obes. Relat. Metab. Disord., 24, 1131-1138.

18. Cho, E.J., Rahman, M.A., Kim, S.W., Baek, Y.M., Hwang, H.J., Oh, J.Y., Hwang, H.S., Lee, S.H. and Yun, J.W. (2008) 
Chitosan oligosaccharides inhibit adipogenesis in 3T3-L1 adipocytes. J. Microbiol. Biotechnol., 18, 80-87.

19. Koyama, Y. and Kusubata, M. (2013) Effects of collagen peptide ingestion on blood lipids in rats fed a high-lipid and high-sucrose diet. Food Sci. Technol. Res., 19, 1149-1153.

20. An, S.M., Seong, K.Y., Yim, S.G., Hwang, Y.J., Bae, S.H., Yang, S.Y. and An, B.S. (2018) Intracutaneous delivery of gelatins induces lipolysis and suppresses lipogenesis of adipocytes. Acta Biomaterialia, 67, 238-247.

21. Manstein, D., Laubach, H., Watanabe, K., Farinelli, W., Zurakowski, D. and Anderson, R.R. (2008) Selective cryolysis: a novel method of non-invasive fat removal. Lasers Surg. Med., 40, 595-604.

22. Moreno-Moraga, J., Valero-Altes, T., Riquelme, A.M., Isarria-Marcosy, M.I. and de la Torre, J.R. (2007) Body contouring by non-invasive transdermal focused ultrasound. Lasers Surg. Med., 39, 315-323.

23. Mulholland, R.S., Paul, M.D. and Chalfoun, C. (2011) Noninvasive body contouring with radiofrequency, ultrasound, cryolipolysis, and low-level laser therapy. Clin. Plast. Surg., 38, 503-520.

24. Yang, S.Y., O'Cearbhaill, E.D., Sisk, G.C., Park, K.M., Cho, W.K., Villiger, M., Bouma, B.E., Pomahac, B. and Karp, J.M. (2013) A bio-inspired swellable microneedle adhesive for mechanical interlocking with tissue. Nat. Commun., 4 , 1702.

25. Lanthier, N. and Leclercq, I.A. (2014) Adipose tissues as endocrine target organs. Best Pract. Res. Clin. Gastroenterol., 28, 545-558.

26. Weisberg, S.P., McCann, D., Desai, M., Rosenbaum, M., Leibel, R.L. and Ferrante, A.W., Jr. (2003) Obesity is associated with macrophage accumulation in adipose tissue. $J$. Clin. Invest., 112, 1796-1808.

27. Weyer, C., Foley, J.E., Bogardus, C., Tataranni, P.A. and Pratley, R.E. (2000) Enlarged subcutaneous abdominal adipocyte size, but not obesity itself, predicts type II diabetes independent of insulin resistance. Diabetologia, 43, 1498-
1506.

28. van der Maaden, K., Jiskoot, W. and Bouwstra, J. (2012) Microneedle technologies for (trans)dermal drug and vaccine delivery. J. Control. Release, 161, 645-655.

29. Bachert, C., Mannent, L., Naclerio, R.M., Mullol, J., Ferguson, B.J., Gevaert, P., Hellings, P., Jiao, L., Wang, L., Evans, R.R., Pirozzi, G., Graham, N.M., Swanson, B., Hamilton, J.D., Radin, A., Gandhi, N.A., Stahl, N., Yancopoulos, G.D. and Sutherland, E.R. (2016) Effect of subcutaneous dupilumab on nasal polyp burden in patients with chronic sinusitis and nasal polyposis: a randomized clinical trial. JAMA, 315, 469-479.

30. Ratnayake, W.M., Sarwar, G. and Laffey, P. (1997) Influence of dietary protein and fat on serum lipids and metabolism of essential fatty acids in rats. Br. J. Nutr., 78, 459-467.

31. de Ferranti, S. and Mozaffarian, D. (2008) The perfect storm: obesity, adipocyte dysfunction, and metabolic consequences. Clin. Chem., 54, 945-955.

32. Mighiu, P.I., Yue, J.T., Filippi, B.M. and Lam, T.K. (2012) Hypothalamic glucagon signaling regulates glucose production. Diabetes, 61, A55.

33. Kang, E.-J., Lee, J.-E., An, S.-M., Lee, J.H., Kwon, H.S., Kim, B.C., Kim, S.J., Kim, J.M., Hwang, D.Y. and Jung, Y.-J. (2015) The effects of vitamin D3 on lipogenesis in the liver and adipose tissue of pregnant rats. Int. J. Mol. Med., 36, 1151-1158.

34. Horton, J.D., Shimomura, I., Brown, M.S., Hammer, R.E., Goldstein, J.L. and Shimano, H. (1998) Activation of cholesterol synthesis in preference to fatty acid synthesis in liver and adipose tissue of transgenic mice overproducing sterol regulatory element-binding protein-2. J. Clin. Invest., 101, 2331-2339.

35. Shimano, H., Horton, J.D., Shimomura, I., Hammer, R.E., Brown, M.S. and Goldstein, J.L. (1997) Isoform 1c of sterol regulatory element binding protein is less active than isoform $1 \mathrm{a}$ in livers of transgenic mice and in cultured cells. $J$. Clin. Invest., 99, 846-854. 